\title{
Ansiedade Social na Infância e Pré-Adolescência: Adaptação para o Português de Portugal da SASC-R
}

\author{
Social Anxiety Disorder in Childhood and Pre-Adolescence: Adaptation \\ of the SASC-R for European Portuguese
}

\author{
Ana Cristina Martins ${ }^{*}, a$, J. Paulo Almeida ${ }^{b, c} \&$ Victor Viana $^{b, d}$ \\ ${ }^{a}$ Instituto Superior de Ciências da Saúde - Norte, Gandra, Concelho de Paredes, Portugal, \\ ${ }^{b}$ Hospital S. João, Porto, Distrito de Porto, Portugal, \\ 'Instituto Superior da Maia, Avioso São Pedro, Concelho de Maia, Portugal \\ $\&{ }^{d}$ Universidade do Porto, Porto, Distrito de Porto, Portugal
}

\begin{abstract}
Resumo
A Escala de Ansiedade Social para Crianças (forma revista) - SASC-R destina-se a avaliar as experiências de ansiedade social e de evitamento das crianças e pré-adolescentes no contexto das relações com os pares. Neste estudo, pretende-se validar para a população portuguesa a SASC-R, utilizando a estrutura proposta pelos autores da escala original, através da análise fatorial confirmatória (AFC). Procedemos à aplicação da SASC-R numa amostra de 486 crianças entre os 9 e os 15 anos. Os resultados indicam que a escala replica os fatores da versão original, possui boa consistência interna e uma validade de constructo bastante satisfatória. Este estudo sugere que a SASC-R é uma escala útil na avaliação da ansiedade social em crianças.

Palavras-chave: Ansiedade social, fobia social, avaliação psicológica, crianças/pré-adolescentes, validação.
\end{abstract}

\begin{abstract}
The Social Anxiety Scale for Children (revised form) - SASC-R evaluates the experience of social anxiety and avoidance felt by children and pre-adolescents in the context of relationship with their peers. This study aims to validate the Portuguese version of the SASC-R by confirmatory factor analysis using the structure proposed by the authors of the original scale. In a survey donewith 486 children between the ages of nine and 15 years, the results show that the scale reproduces the original factors, has a good internal consistency and a quite satisfactory construct validity. This study suggests that the SASC-R is a useful scale on the evaluation of social anxiety among children.

Keywords: Social anxiety, social phobia, psychological evaluation, children/pre-teen, validation.
\end{abstract}

A Fobia Social (FS) representa um problema grave de saúde mental com características incapacitantes nas suas diferentes formas de apresentação. A mais comum é o medo exacerbado de exposição à avaliação por parte dos outros e de ser humilhado ou ridicularizado em situações sociais por apresentar atitudes inadequadas (Casas, Álvarez, Lopez, \& Aranda, 2012; Ito, Roso, Tiwari, Kendall, \& Asbahr, 2008; Pinto Gouveia, 2000). A exposição a estas situações desencadeia uma marcada sintomatologia dos três sistemas de resposta (cognitivo, fisiológico e motor) levando ao desconforto e evitamento (Ost, 2008; Silveira \& Valença, 2005; Toit \& Stein, 2003).

$\mathrm{Na}$ infância e na adolescência, a fobia social está associada a interferências significativas ao nível social,

\footnotetext{
* Endereço para correspondência: Instituto Superior de Ciências da Saúde - Norte, Rua Central de Gandra, 1317, Vilarinho de Cima, Gandra, Portugal 4585-116. E-mail: anaazevedo.martins@gmail.com
}

académico e ocupacional, conduzindo a uma qualidade de vida reduzida (Salvador, 2009). As crianças e adolescentes com elevada ansiedade social têm menos amigos, sentem-se mais sozinhas e solitárias (Albano, Chorpita, \& Barlow, 2003; Beidel, Turner, \& Morris, 1999; Beidel et al., 2007), e apresentam menores níveis de aceitação e suporte no grupo de pares, sendo mais suscetíveis de serem ignoradas, rejeitadas ou ridicularizadas (Blote, Kint, \& Westenberg, 2007; Greco \& Morris, 2005; Storch \& Masia-Warner, 2004). Este fenómeno é importante sobretudo por esta ser uma idade critica para a socialização e desenvolvimento das relações e habilidades interpessoais (Salvador, 2009; Sandín, Chorot, Valiente, Santed, \& Sánchez-Arribas, 1999). Apresentam uma baixa perceção das suas capacidades cognitivas, elevados níveis de ansiedade nas situações de testes e atividades escolares, traduzindo-se na diminuição do rendimento escolar esperado e até mesmo numa recusa em ir à escola (Cunha \& Salvador, 2000; Stein \& Kean, 2000; Van Ameringen, Mancini, \& Farvolden, 2003). 
Martins, A. C., Almeida, J. P. \& Viana, V. (2014). Ansiedade Social na Infância e Pré-Adolescência: Adaptação para o Português de Portugal da SASC-R.

Uma avaliação compreensiva e rigorosa da fobia social em crianças e adolescentes requer uma abordagem abrangente, que envolva múltiplas fontes de informação, múltiplos contextos e múltiplos tipos de instrumentos de avaliação (Cunha \& Pinto Gouveia, 1997; Cunha \& Salvador, 2000). Os questionários de auto-resposta, cuja finalidade é identificar sintomas e comportamentos específicos relacionados com a ansiedade social, e quantificar a sua ocorrência ou intensidade, são instrumentos extremamente úteis para avaliar a experiência de ansiedade, segundo a perspetiva da criança ou do adolescente (Salvador, 2009). No entanto, focando a nossa atenção nos questionários que procuram avaliar especificamente a fobia social na infância e adolescência, deparamo-nos com uma área menos desenvolvida do que a avaliação deste quadro clínico em adultos. De seguida, fazemos uma descrição breve de alguns dos instrumentos mais amplamente divulgados na avaliação da fobia social na infância e adolescência.

A Escala de Medo de Avaliação Negativa (FNE) e a Escala de Evitamento e Desconforto Sociais (SAD; Watson \& Friend, 1969), inicialmente construídas para adultos, apresentaram uma elevada consistência interna $(\alpha=0,94)$, mostrando o SAD uma estabilidade temporal de 0,68 e o FNE de 0,78 . No entanto, têm sido também estudadas já em populações de adolescentes (Garcia-López, Olivares, Hidalgo, Beidel, \& Turner, 2001; Warren, Good, \& Velten, 1984). O SAD é composto por 28 itens que avaliam a experiência de mal-estar, desconforto e ansiedade em situações sociais, bem como, o evitamento deliberado dessas situações. O FNE, formado por 30 itens, procura medir a expectativa, o medo da avaliação negativa pelos outros e os evitamentos de situações avaliativas. A Escala Revista de Ansiedade Social para Crianças (SASC-R Social Anxiety Scale for Children-Revised; La Greca \& Stone, 1993) procura avaliar a experiência subjetiva de ansiedade social em crianças. É breve, fácil de administrar e possui boas propriedades psicométricas. Esta foi uma das escalas que utilizámos na presente investigação. A Escala de Ansiedade Social para Adolescentes (SAS-A - Social Anxiety Scale for Adolescents; La Greca \& Lopez, 1998) é uma adaptação para a população adolescente da SASC-R (La Greca \& Stone, 1993). É uma escala de auto-resposta com 22 itens (18 relacionados com ansiedade e 4 neutros) e à semelhança da SASC-R, revelou boas qualidades psicométricas e uma estrutura fatorial idêntica, constituída pelos mesmos três fatores ou subescalas (La Greca \& Lopez, 1998; La Greca \& Stone, 1993). A versão Portuguesa da SAS-A foi estudada por Cunha e colaboradores (Cunha, Pinto Gouveia, Alegre, \& Salvador, 2004), numa amostra de 522 jovens entre os 12 e os 18 anos, do distrito de Coimbra. A análise fatorial revelou uma estrutura de três fatores semelhante à encontrada para a versão americana (La Greca \& Lopez, 1998), embora se tivessem encontrado algumas diferenças nos itens que os constituem. A versão Portuguesa da SAS-A revelou possuir valores elevados de consistência interna, tanto para o total da escala como para as subescalas, indicando uma boa fidelidade ( $\alpha=0,88$ para o Total da Escala, $\alpha=0,87$ para o fator FNE, $\alpha=0,74$ para o fator SAD-N e $\alpha=0,71$ para o fator SAD-G). A estabilidade temporal ( 1 mês) mostrou resultados aceitáveis $(r=0,74)$. O Inventário de Ansiedade e Fobia Social para Crianças (SPAI-C - Social Phobia and Anxiety Inventory for Children; Beidel, Turner, \& Morris, 1995) que procura avaliar a fobia social segundo os critérios do DSM-IV (American Psychiatric Association [APA], 1994), em crianças e adolescentes com idades compreendidas entre os 9 e os 14 anos. Este foi também um dos instrumentos utilizados neste trabalho. O Inventário de Ansiedade e Fobia Social (SPAI - Social Phobia and Anxiety Inventory; Beidel, Turner, Stanley, \& Dancu, 1989), um questionário desenvolvido para adultos com fobia social e validado para a população adolescente (Clark et al., 1994; Garcia-López et al., 2001; Olivares, Garcia-López, Hidalgo, Turner, \& Beidel, 1999), que avalia medos sociais e ansiedade. É constituído por 32 itens que medem componentes somáticos, cognitivos, comportamentais e de evitamento associados à ansiedade social, e por 13 itens que avaliam vários aspetos de agorafobia. Reporta elevada consistência interna para as escalas de fobia social e de agorafobia $(\alpha=0,96$ e 0,85 , respetivamente), e uma boa consistência teste-reteste ( $r=$ 0,86 e $r=0,85$, respetivamente). O Inventário de Fobia Social (SPIN - Social Phobia Inventory; Connor et al., 2000; Johnson, Inderbitzen-Nolan, \& Anderson, 2006) é constituído por 17 itens que avaliam o medo, o evitamento e os sintomas fisiológicos associados à ansiedade social. $\mathrm{O}$ SPIN demonstrou qualidades psicométricas satisfatórias, quer ao nível da fidelidade teste-reteste, quer ao nível da consistência interna $(\alpha=0,87$ a $\alpha=0,94)$. A análise fatorial do instrumento revelou a existência de cinco fatores: (a) falar com estranhos e situações sociais; (b) crítica e embaraço; (c) alterações fisiológicas; (d) figuras de autoridade; (e) evitar ser o centro das atenções e discursar em público. O Inventário de Fobia Social (SoPhI - Social Phobia Inventory; Moore \& Gee, 2003), constituído por 21 itens, avalia a ansiedade social segundo os critérios do DSM-IV (APA, 1994) e inclui um item adicional que avalia se os sintomas de ansiedade social foram experienciados por um período superior a seis meses. A análise fatorial do SoPhI revelou a existência de um só fator que explica aproximadamente $59 \%$ da variância e que demonstra uma boa consistência interna $(\alpha=0,93)$. Em Portugal, foi desenvolvida a Escala de Ansiedade e Evitamento de Situações Sociais para Adolescentes (EAESSA; Cunha, Pinto Gouveia, Salvador, \& Alegre, 2004) para avaliar aspetos de ansiedade e evitamento de adolescentes com fobia social. É formada por 34 situações sociais e constituída por duas subescalas, a subescala de desconforto/ansiedade e a subescala de evitamento. A EAESSA revelou valores elevados de consistência interna para ambas as subescalas $(\alpha=0,91$ para a subescala de desconforto/ansiedade e $\alpha=0,87$ para a subescala de evitamento) e boa fidelidade teste-reteste num intervalo de 5 semanas $(r=0,74, p<0,01$ para a subescala de desconforto/ansiedade e $r=0,71, p<0,01$ para a subescala de evitamento). 
Outros questionários que avaliam constructos associados à ansiedade social podem também ser utilizados: Perfil de Perceção de Si Mesmo para Crianças (SPPC - Self-Perception Profile for Children; Harter, 1982), Avaliação de Aptidões Sociais de Matson (MESSY - Matson Evaluation of Social Skills with Youngsters; Matson, Rotatori, \& Helsel, 1983), e o Inventário de Aptidões Sociais de Adolescentes (TISS - Teenage Inventory of Social Skills; Inderbitzen \& Foster, 1992).

Tendo em conta a escassez de instrumentos de medida disponíveis para avaliar crianças e adolescentes com ansiedade social elevada em Portugal, o presente estudo teve como objetivo validar a SASC-R para a população portuguesa, recorrendo a uma amostra específica de crianças e pré-adolescentes do ensino regular, testando a estrutura proposta pelos autores da escala original através da análise fatorial confirmatória (AFC). Paralelamente procurou-se analisar a validade convergente e discriminativa da escala.

\section{Metodologia}

\section{Participantes}

A amostra é constituída por 486 estudantes do $2^{\circ}$ e $3^{\circ}$ ciclo de três escolas públicas da zona do grande Porto, sendo que 248 (51\%) são do sexo masculino e 238 (49\%) do sexo feminino. Relativamente à escolaridade $(M=6,79$; $D P=1,35$ ), no $2^{\circ}$ ciclo encontram-se 224 sujeitos (124 rapazes e 100 raparigas) e no $3^{\circ}$ ciclo, 162 elementos (124 rapazes e 138 raparigas). A distribuição dos alunos por anos de escolaridade encontra-se da seguinte forma: 103 alunos no $5^{\circ}$ ano $(21,2 \%), 121$ alunos no $6^{\circ}$ ano $(24,9 \%)$, 113 alunos no $7^{\circ}$ ano $(23,3 \%), 75$ alunos no $8^{\circ}$ ano $(15,4 \%)$ e 74 alunos no $9^{\circ}$ ano $(15,2 \%)$. As idades estão compreendidas entre os 9 e 15 anos $(M=12,05 ; D P=1,63)$ estando os sujeitos distribuídos de forma homogénea ao longo do intervalo estabelecido.

\section{Instrumentos}

A SASC-R (La Greca \& Stone,1993) é composta por 22 itens de auto-relato ( 18 itens relacionados com estados de ansiedade e 4 itens adicionais), avaliados numa escala tipo Likert de cinco pontos $(1=$ nunca até $5=$ sempre $)$. A análise fatorial da SASC-R identificou três fatores que correspondem a três subescalas: Medo de Avaliação Negativa (FNE - Fear of Negative Evaluation), Desconforto e Evitamento Social em Situações Novas (SAD- New - Social Avoidance and Distress Specific to New Situations) e Desconforto e Evitamento Social Geral (SAD-G - Generalized Social Avoidance and Distress). A subescala FNE reflete o medo e a preocupação da criança, relativamente à possibilidade de uma avaliação negativa por parte dos outros. A subescala SAD-N é formada por itens que avaliam o desconforto e a ansiedade em situações sociais novas ou com crianças desconhecidas. Finalmente, a SAD-G avalia a ansiedade e inibição em situações sociais gerais, incluindo pessoas conhecidas. A SASC-R revelou uma boa consistência interna (FNE $\alpha=0,86$, SAD-N $\alpha=0,78$ e SAD-G $\alpha=0,69$ ), boa fidelidade teste-reteste, boa validade concorrente e boa validade discriminativa. As correlações inter-escalas têm revelado que as subescalas da SASC-R estão relacionadas, mas medem constructos de ansiedade social distintos.

Para se estudar a validade concorrente da SASC-R, utilizou-se o SPAI-C (Beidel et al., 1995). É um instrumento composto por 26 itens que têm como objectivo medir uma grande variedade de potenciais situações produtoras de ansiedade. $\mathrm{O}$ estudo da análise fatorial revelou a existência de três fatores: Assertividade/Conversação Geral, Encontros Sociais Tradicionais, Performance Pública. Os resultados obtidos apontaram para valores excelentes relativamente à consistência interna $(\alpha=0,95)$, à fidelidade teste-reteste $(0,86$ para duas semanas de intervalo), à validade concorrente e à validade discriminativa. Este questionário discrimina eficazmente crianças com perturbação de ansiedade social de controlos normais (Beidel et al., 1995), assim como, crianças com fobia social daquelas que apresentam desordens externalizadoras (Beidel, Turner, Hamlin, \& Morris, 2000).

\section{Procedimento}

O processo de tradução e adaptação da SASC-R teve como base a orientação proposta por Bradley (1996). Foi feita a tradução pelo investigador e simultaneamente por tradutor independente. Confrontaram-se as versões para elaboração da primeira versão em português, seguida de retroversão por tradutor independente não conhecedor da versão inicial em língua inglesa e novo confronto de versões (original e retrovertida) com o objectivo de avaliar a identidade do conteúdo dos itens. Prosseguiu-se com a administração da escala a 12 pré-adolescentes (pré-teste) para avaliar a adequação e compreensão dos itens da versão experimental e procedeu-se à elaboração das versões a utilizar no estudo.

Foram seleccionadas três escolas públicas urbanas e suburbanas do distrito do Porto para a referida investigação. As turmas foram seleccionadas segundo um processo aleatório. A SASC-R e o SPAI-C foram administrados às turmas nas salas de aula, depois de obtido o consentimento das escolas e dos encarregados de educação após uma explicação acerca dos objectivos, metodologia e utilidade do trabalho de investigação. Os directores de turma tiveram acesso a uma folha de rosto que acompanhava a bateria de questionários, na qual eram explicadas de forma clara e sucinta as instruções para administração, e solicitada a sua supervisão durante a aplicação.

\section{Análise de Dados}

Através do programa estatístico SPSS versão 19.0 para Windows, determinou-se a consistência interna da SASC-R através do coeficiente alpha de Cronbach, para a totalidade dos itens do questionário e para as subescalas que o compõem. Seguidamente, com base na estrutura fatorial proposta pelos autores da escala original, foi realizada a AFC com a amostra de alunos portugueses através do programa Statistica 8.0 (SEPATH). Foram utilizadas medidas 
Martins, A. C., Almeida, J. P. \& Viana, V. (2014). Ansiedade Social na Infância e Pré-Adolescência: Adaptação para o Português de Portugal da SASC-R.

de discrepância e medidas de ajustamento entre os dados e o modelo. Entre as primeiras, recorreu-se à razão entre o chi quadrado e os graus de liberdade $\left(\mathrm{X}^{2} / d f\right)$ e ao root mean square error of approximation (RMSEA). Segundo Oreskong e Sorbom (1993), os valores de $\mathrm{X}^{2} / d f$ deverão situar-se entre 2 e 5 para serem considerados adequados. $\mathrm{O}$ RMSEA, que avalia o grau de discrepância no ajustamento entre a matriz de co-variância do modelo original e da amostra, deverá apresentar valores iguais ou inferiores a 0,05 para um erro de aproximação excelente, no entanto, valores inferiores a 0,08 são já considerados satisfatórios (Cole, 1987). Os índices de ajustamento utilizados foram o comparative fit index (CFI), o goodness of fit índex (GFI), o ajusted GFI (AGFI), o non-normed fit index (NNFI) e o normed fit index (NFI). Para estas medidas os resultados variam entre 0 e 1 , sendo que valores superiores a 0,9 são considerados indicadores de bom ajustamento (Bentler, 1990).

Finalmente, para se determinar a validade convergente da SASC-R averiguou-se a sua correlação com o SPAI-C através do coeficiente de correlação de Pearson. Para analisar a capacidade da escala e subescalas diferenciarem resultados em sub-grupos da amostra, foi realizada a análise da validade discriminativa em relação ao sexo, à idade e ao nível de escolaridade dos participantes, recorrendo-se ao Independent Samples Test (T-Test) e à ANOVA $(F)$.

\section{Resultados}

A partir da análise da consistência interna, foi obtido para o total da escala um alpha de Cronbach de 0,86, conforme se apresenta na Tabela 1. Relativamente aos seus fatores constituintes, os resultados evidenciaram que o factor 1, Medo de Avaliação Negativa (FNE), apresenta um alpha de 0,85 e integra 8 itens, que traduzem uma preocupação com a avaliação negativa por parte dos outros. Neste primeiro fator, as correlações dos itens variaram de 0,36 a 0,71. O fator 2, Desconforto e Evitamento Social em Situações Novas (SAD-N), apresenta uma consistência interna de 0,73 , as correlações dos itens variaram de 0,26 a 0,60 e engloba 6 itens que reflectem preocupação e mal-estar sentido em situações sociais novas. Finalmente,

Tabela 1

Consistência Interna (Alpha de Cronbach) da SASC-R Total

\begin{tabular}{|c|c|c|}
\hline & $\begin{array}{l}\text { Correlação } \\
\text { Item-total }\end{array}$ & $\begin{array}{l}\alpha \text { de Cronbach se } \\
\text { Item Eliminado }\end{array}$ \\
\hline 1-Preocupa-me o ter que fazer coisas novas à frente dos outros colegas & 0,381 & 0,86 \\
\hline 2-Gosto de brincar com os meus colegas & 0,025 & 0,87 \\
\hline 3-Preocupa-me com o facto de poder ser gozado(a) & 0,495 & 0,85 \\
\hline 4-Sinto que sou tímido(a) quando estou com rapazes/raparigas que não conheço & 0,520 & 0,85 \\
\hline 5-Só falo com colegas que eu conheço muito bem & 0,250 & 0,86 \\
\hline 6-Acho que os meus colegas falam de mim nas minhas costas & 0,360 & 0,86 \\
\hline 7-Eu gosto de ler & 0,223 & 0,86 \\
\hline 8-Preocupa-me o que os outros colegas pensam de mim & 0,547 & 0,85 \\
\hline 9-Tenho medo que os outros possam não gostar de mim & 0,641 & 0,85 \\
\hline 10-Fico nervoso(a) quando falo com colegas que não conheço muito bem & 0,572 & 0,85 \\
\hline 11-Gosto de fazer desporto & 0,073 & 0,87 \\
\hline 12-Preocupa-me com o que os outros dizem acerca de mim & 0,584 & 0,85 \\
\hline 13-Fico nervoso quando conheço colegas novos & 0,568 & 0,85 \\
\hline 14-Preocupa-me que os outros colegas não gostem de mim & 0,657 & 0,84 \\
\hline 15-Fico calado(a) quando estou num grupo de rapazes/raparigas & 0,492 & 0,85 \\
\hline 16-Gosto de fazer as coisas sozinho(a) & 0,244 & 0,86 \\
\hline 17-Penso que os outros colegas fazem troça de mim & 0,513 & 0,85 \\
\hline 18-Se discuto com outro colega, preocupa-me que ele/a possa não gostar de mim & 0,574 & 0,85 \\
\hline $\begin{array}{l}\text { 19-Tenho medo de convidar outros(as) rapazes/raparigas para fazer qualquer coisa } \\
\text { comigo, porque podem dizer que não }\end{array}$ & 0,532 & 0,85 \\
\hline 20-Sinto-me nervoso(a) quando estou perto de alguns/algumas colegas & 0,452 & 0,85 \\
\hline 21-Sinto que sou tímido(a) até com colegas que conheço bem & 0,470 & 0,85 \\
\hline 22-Para mim é dificil pedir a outros colegas para fazer coisas comigo & 0,472 & 0,85 \\
\hline
\end{tabular}


o terceiro factor, Desconforto e Evitamento Social Geral (SAD-G), constituído por 4 itens com correlações que variaram de 0,46 a 0,53, evidencia um alpha de 0,70 e diz respeito ao evitamento e ansiedade provocados por situações sociais gerais.

Em termos de validade de construto, os resultados obtidos através da AFC (Tabela 2) sugerem que o modelo original da SASC-R indicado por La Greca e Stone (1993), demonstrou uma adequabilidade satisfatória, apresentando todas as medidas de discrepância e de ajustamento valores próximos dos recomendados. Mais especificamente, os valores de $X^{2} / d f(3,70)$, RMSEA $(0,08)$, CFI $(0,90)$, GFI $(0,90)$, AGFI $(0,90)$, NFI $(0,83)$ e NNFI $(0,90)$, indicam que o constructo subjacente à construção da SASC-R se adequa à nossa amostra. De salientar, que à exceção do NFI, que é o único índice que não vai de encontro aos resultados desejados mas que revela uma aproximação satisfatória, todos os outros critérios são preenchidos pois situam-se dentro dos valores de referência.

Tabela 2

Índices de Ajustamento Obtidos pela AFC

\begin{tabular}{ccc}
\hline & $\begin{array}{c}\text { Valores } \\
\text { recomendados }\end{array}$ & Escala SASC-R \\
\hline $\mathrm{X}^{2} /$ & & 488,6 \\
$d f$ & & 132 \\
$\mathrm{X}^{2} / d f$ & {$[2-5]$} & 3,70 \\
$\mathrm{RMSEA}$ & 0,08 & 0,08 \\
$\mathrm{CFI}$ & 0,90 & 0,90 \\
$\mathrm{GFI}$ & 0,90 & 0,90 \\
$\mathrm{AGFI}$ & 0,90 & 0,90 \\
$\mathrm{NNFI}$ & 0,90 & 0,90 \\
NFI & 0,90 & 0,83 \\
\hline
\end{tabular}

Nota. RMSEA: Erro de aproximação à medida; CFI: Índice de ajustamento comparativo; GFI: Índice de bondade do ajustamento; AGFI: Índice de bondade do ajustamento ajustado; NNFI: Índice ajustado não normativo; NFI: Índice ajustado normativo.

A validade convergente do construto foi determinada através da correlação entre a SASC-R e o SPAI-C. Para tal, calculou-se o coeficiente de correlação produto $\mathrm{x}$ momento de Pearson e como se verifica na Tabela 3, os valores totais obtidos na SASC-R correlacionam-se de forma significativa com os resultados totais obtidos no SPAI-C ( $r=0,62$; $p<0,001)$. Também, os resultados obtidos sugerem que os três factores que compõem a SASC-R se encontram correlacionados com o valor total do SPAI-C: FNE ( $r=$ $0,45 ; p<0,001)$; SAD-N $(r=0,64 ; p<0,001)$; SAD-G $(r=0,62 ; p<0,001)$. Os coeficientes de correlação obtidos entre as subescalas da SASC-R (Tabela 3), sugerem que estas embora inter-relacionadas, representam constructos distintos da ansiedade social. De um modo geral, a SASC-R apresenta uma validade convergente satisfatória.
Tabela 3

Validade Concorrente da SASC-R com o SPAI-C

\begin{tabular}{ccccc}
\hline & SPAI-C & Total & FNE & SAD-N \\
\hline SPAI-C & - & & & \\
SASC-R & $0,62^{*}$ & - & & \\
FNE & $0,45^{*}$ & $0,89^{*}$ & - & \\
SAD-N & $0,64^{*}$ & $0,78^{*}$ & $0,52^{*}$ & - \\
SAD-G & $0,62^{*}$ & $0,77^{*}$ & $0,51^{*}$ & $0,65^{*}$ \\
\hline
\end{tabular}

${ }^{*} p<0,001$.

Em termos de validade discriminativa apreciou-se o efeito do sexo quer em relação ao total da escala, quer relativamente aos seus fatores constituintes. Como se pode ver na Tabela 4, os resultados encontrados sugerem que o sexo feminino apresenta valores significativamente superiores aos do sexo masculino, quer em relação ao total da escala $\left(t_{(438)}=-4,83 ; p<0,001\right)$, quer em relação às subescalas FNE $\left(t_{(459)}=-4,11 ; p<0,001\right)$, SAD-N $\left(t_{(465)}=-4,43, p<\right.$ $0,001)$ e SAD-G $\left(t_{(479)}=-2,80 ; p<0,01\right)$.

De seguida, realizou-se um estudo estatístico comparativo entre a SASC-R Total, os seus diferentes factores e o nível etário dos participantes. As idades foram distribuídas por três grupos: o grupo 1 (9 e 10 anos), o grupo 2 (11, 12 e 13 anos) e o grupo 3 ( 14 e 15 anos). Tal como se pode verificar na Tabela 4 , foram encontradas diferenças significativas apenas entre o grupo $2(M=49,79 ; D P=13,37) \mathrm{e}$ o grupo $1(M=45,95 ; D P=12,59)$ na SASC-R total $\left(F_{(439)}\right.$ $=3,13 ; p<0,05)$, e na subescala FNE $\left(F_{(460)}=3,39 ; p<\right.$ $0,05)$ entre os mesmos grupos: o grupo $2(M=22,93 ; D P=$ $7,21)$ e o grupo $1(M=20,72 ; D P=7,25)$.

Finalmente, realizou-se uma análise sobre os efeitos da escolaridade quer em relação ao total da escala, quer relativamente aos seus fatores constituintes. Os anos de escolaridade foram distribuídos por dois grupos: grupo 1 (alunos do $2^{\circ}$ ciclo: $5^{\circ}$ e $6^{\circ}$ ano) e grupo 2 (alunos do $3^{\circ}$ ciclo: $7^{\circ}, 8^{\circ}$ e $9^{\circ}$ ano). No entanto, não foram encontradas diferenças significativas entre os dois grupos de escolaridade na SASC-R total, bem como, nas subescalas FNE, SAD-N e SAD-G. Contudo, uma comparação entre as médias dos diversos grupos de escolaridade permitiu verificar que é o grupo 2 que apresenta valores mais elevados no total da escala.

\section{Discussão}

É amplamente conhecido o facto das experiências de ansiedade social serem frequentes na infância e na adolescência, podendo ter sérias consequências no caso de serem intensas e persistentes. A sua avaliação torna-se assim, um aspecto crucial para a investigação e prática clínica. Neste processo de avaliação, os questionários de auto-resposta desempenham um papel importante permitindo, com facilidade e, de forma fidedigna e válida, ter acesso a formas 
Martins, A. C., Almeida, J. P. \& Viana, V. (2014). Ansiedade Social na Infância e Pré-Adolescência: Adaptação para o Português de Portugal da SASC-R.

Tabela 4

Resultados do Total e Sub-Escalas da SASC-R por Sexo, Idade e Escolaridade

\begin{tabular}{|c|c|c|c|c|c|c|c|c|c|c|c|c|}
\hline & \multicolumn{12}{|c|}{ Sub-escalas SASC-R } \\
\hline & \multicolumn{3}{|c|}{ Total } & \multicolumn{3}{|c|}{ FNE } & \multicolumn{3}{|c|}{ SAD-N } & \multicolumn{3}{|c|}{ SAD-G } \\
\hline & $n$ & $M$ & $D P$ & $n$ & $M$ & $D P$ & $n$ & $M$ & $D P$ & $n$ & $M$ & $D P$ \\
\hline \multicolumn{13}{|l|}{ Sexo } \\
\hline Masculino & 224 & 45,66 & 12,40 & 236 & 20,98 & 6,99 & 237 & 14,48 & 4,49 & 244 & 8,01 & 3,12 \\
\hline Feminino & 216 & 51,49 & 12,92 & 225 & 23,68 & 7,10 & 230 & 16,32 & 4,46 & 237 & 8,84 & 3,39 \\
\hline$t$ & & $-4,83$ & & & $-4,11$ & & & $-4,43$ & & & $-2,80$ & \\
\hline$g l$ & & 438 & & & 459 & & & 465 & & & 479 & \\
\hline$p$ & & 0,000 & & & 0,000 & & & 0,000 & & & 0,005 & \\
\hline \multicolumn{13}{|l|}{ Idades } \\
\hline Grupo 1 & 91 & $45,95^{*}$ & 12,59 & 97 & $20,72^{*}$ & 7,25 & 98 & 15,47 & 4,65 & 98 & 8,10 & 3,15 \\
\hline Grupo 2 & 243 & $49,79^{*}$ & 13,37 & 256 & $22,93^{*}$ & 7,21 & 259 & 15,50 & 4,47 & 270 & 8,66 & 3,26 \\
\hline Grupo 3 & 106 & 47,83 & 12,09 & 108 & 22,20 & 6,83 & 110 & 15,04 & 4,72 & 113 & 8,13 & 3,41 \\
\hline$F$ & & 3,13 & & & 3,39 & & & 0,421 & & & 1,69 & \\
\hline$g l$ & & 439 & & & 460 & & & 466 & & & 480 & \\
\hline \multicolumn{13}{|l|}{ Escolaridade } \\
\hline Grupo 1 & 203 & 48,19 & 13,19 & 213 & 21,92 & 7,34 & 216 & 15,58 & 4,67 & 222 & 8,43 & 3,26 \\
\hline Grupo 2 & 237 & 48,81 & 12,80 & 248 & 22,62 & 7,03 & 251 & 15,22 & 4,48 & 259 & 8,41 & 3,30 \\
\hline$t$ & & $-0,50$ & & & $-1,05$ & & & 0,85 & & & 0,06 & \\
\hline$g l$ & & 438 & & & 459 & & & 465 & & & 479 & \\
\hline$p$ & & 0,619 & & & 0,296 & & & 0,397 & & & 0,949 & \\
\hline
\end{tabular}

${ }^{*} p<0,05$.

de pensar, sentir e agir das crianças e adolescentes (Cunha, Pinto Gouveia, Alegre, et al., 2004).

O presente estudo teve como principal objetivo adaptar para a população portuguesa um instrumento de avaliação da ansiedade social destinado a crianças e pré-adolescentes. Para tal, recorreu-se à SASC-R pois através de diversas investigações, esta escala tem sempre revelado boas características psicométricas e utilidade no estudo da ansiedade social nas crianças e pré-adolescentes no contexto de interação com os pares (Kuusikko et al., 2009; La Greca \& Stone, 1993; Reijntjes, Dekovic, Telch, \& Michael, 2007; Sandín et al., 1999).

No que respeita às análises psicométricas da SASC-R para a versão portuguesa, os resultados da $\mathrm{AFC}$ indicaram que o modelo composto por três fatores sugerido por $\mathrm{La}$ Greca e Stone (1993), preenche praticamente todos os critérios de ajustamento para a validação do constructo. Esta interpretação é apoiada pelos valores dos índices de adequabilidade obtidos, que se situam dentro dos valores de referência ou então são muito aproximados. A versão portuguesa da SASC-R mostrou também possuir valores elevados de consistência interna para o total e subescalas, indicando uma boa fidelidade. Relativamente à validade concorrente, a SASC-R revelou correlações moderadas com o SPAI-C, no entanto, esperavam-se correlações mais significativas já que são instrumentos que avaliam o mesmo constructo. Os valores moderados das correlações entre as subescalas da SASC-R sugerem que estas, embora inter-relacionadas representam constructos distintos da ansiedade social.

No que se refere à validade discriminativa, os valores obtidos para o total e subescalas da SASC-R na população portuguesa foram semelhantes aos encontrados na população americana (La Greca \& Stone, 1993). A mesma consistência de dados verificou-se em relação à distribuição da ansiedade social segundo o sexo, constatando-se que à semelhança dos estudos americanos, as raparigas apresentam valores significativamente mais elevados que os rapazes quer para o total quer para as sub-escalas da SASC-R. Em relação ao papel da idade na ansiedade social, 
também se verificaram diferenças significativas entre os grupos na população portuguesa, parecendo haver uma menor ansiedade social, bem como, um menor receio de avaliação negativa no grupo de crianças mais novas $(9$ e 10 anos). Os valores mais elevados foram obtidos nas idades intermédias (11, 12 e 13 anos), a que se seguiu uma ligeira diminuição com o início da adolescência (14 e 15 anos). Estes resultados estão de acordo com alguns estudos realizados na população americana com crianças e adolescentes (Beidel et al., 1995), e poderão ser explicados pelos diferentes desafios desenvolvimentais colocados pelos diferentes desafios desenvolvimentais colocados a essa a essa faixa etária. Ao longo da infância, a integração social e adaptação à escola desempenham um papel importante no desenvolvimento global, e à medida que as crianças se tornam mais velhas a aceitação pelo grupo de pares torna-se determinante. Tendo em conta que esta aceitação depende da forma particular como o indivíduo é percebido, é nesta fase que as crianças tomam consciência da importância que as impressões sociais podem causar nos outros. À medida que vão ficando mais velhas, as rápidas transformações corporais e o desempenho de novos papéis contribuem para um aumento da insegurança e para o medo de não conseguir causar a impressão desejada. Paralelamente a estas mudanças, os pais e colegas tornam-se menos tolerantes perante comportamentos pouco adequados, aumentando assim, a possibilidade de serem criticadas ou ridicularizadas. Neste contexto, percebe-se que esta fase da pré-adolescência e adolescência apareça como um dos períodos de vida especialmente relevante para o aparecimento de ansiedade social.

Assim, de uma forma geral verifica-se que a SASC-R é capaz de discriminar o efeito do sexo e da idade em relação à ansiedade social. No entanto, para o grau de escolaridade os resultados não foram significativamente diferentes entre os grupos da nossa amostra. Porém, a partir de uma análise informal dos dados obtidos verificou-se alguma discrepância comparativamente aos resultados da população americana, que serve de base para a construção deste instrumento. Seria de esperar que as crianças em níveis de escolaridade mais baixos revelassem mais ansiedade social do que as crianças em níveis de escolaridade superiores ( $\mathrm{La}$ Greca \& Stone, 1993). Contudo, uma comparação entre as médias dos dois grupos de escolaridade na população portuguesa, permitiu verificar que é o grupo $2\left(3^{\circ}\right.$ ciclo: $7^{\circ}, 8^{\circ}$ e $9^{\circ}$ ano $)$ e não o grupo $1\left(2^{\circ}\right.$ ciclo: $5^{\circ}$ e $6^{\circ}$ ano $)$ que apresenta valores mais elevados no total da escala, ou seja, são as crianças em níveis de escolaridade superiores que apresentam mais ansiedade. Tendo em conta que o terceiro ciclo do ensino básico corresponde a um período de transição para o ensino secundário, este dado pode sugerir, apesar de não haver diferenças significativas, que nesta fase possa haver um aumento da ansiedade social pelas mudanças e adaptações que implica, nomeadamente, a mudança de escola, novas deslocações, o confronto com um ambiente físico e social mais amplo, novos colegas e novos métodos de ensino. Futuras investigações poderão contribuir para uma clarificação deste aspecto.

Algumas limitações podem ser apontadas a este estudo. A primeira prende-se com a ausência de resultados acerca da estabilidade temporal da medida, tornando-se necessário a realização de estudos sobre a fidelidade do questionário por meio de teste-reteste. Outra limitação está relacionada com a natureza não clínica da amostra. Estudos futuros deverão analisar o comportamento deste instrumento com amostras clínicas nomeadamente, a sua capacidade para discriminar populações normais de populações referenciadas para os serviços de saúde e/ou que revelem perturbações de ansiedade social conforme os critérios de diagnóstico vigentes. É também relevante replicar estes dados com amostras maiores e mais diversificadas do ponto de vista geográfico. São necessárias novas investigações que analisem a validade discriminativa da SASC-R para garantir a sua utilidade e relevância clínica. Investigações futuras poderão complementar esta análise factorial com estudos de validade de critério concorrente, aplicando outros instrumentos paralelos já adaptados ao contexto português.

Não obstante as limitações apontadas, os resultados deste estudo revelam que a versão portuguesa da SASC- $\mathrm{R}$ apresenta características psicométricas satisfatórias e que este é um instrumento a ter em conta na avaliação da ansiedade social nas crianças e pré-adolescentes.

\section{Referências}

Albano, A., Chorpita, B., \& Barlow, D. (2003). Childhood anxiety disorders. In E. Mash \& R. Barkley (Eds.), Child psychopathology (pp. 279-371). New York: Guilford Press.

American Psychiatric Association. (1994). Diagnostic and statistical manual of mental disorders: DSM-IV ( $4^{\text {th }}$ ed.). Washington, DC: Author.

Beidel, D., Turner, S., Hamlin, K., \& Morris, T. (2000). The Social Phobia Inventory for Children (SPAI-C): External and discriminative validity. Behavior Therapy, 31(1), 75-87.

Beidel, D. C., Turner, S. M., \& Morris, T. L. (1995). A new inventory to assess childhood social anxiety and phobia: The Social Phobia and Anxiety Inventory for Children. Psychological Assessment, 7(1), 73-79.

Beidel, D. C., Turner, S. M., \& Morris, T. L. (1999). Psychopathology of childhood social phobia. Journal of the American Academy of Child and Adolescent Psychiatry, 38, 643-650.

Beidel, D. C., Turner, S. M., Stanley, M., \& Dancu, C. (1989). The Social Phobia and Anxiety Inventory. Behavior Therapy, 20, 417-427.

Beidel, D. C., Turner, S. M., Young, B. J., Ammerman, R. T., Sallee, F. R., \& Crosby, L. (2007). Psychopathology of adolescent social phobia. Journal of Psychopathology and Behavioral Assessment, 29, 47-54.

Bentler, P. (1990). Comparative fit indexes in structural models. Psychological Bulletin, 107(2), 238-246.

Blote, A. W., Kint, M. J., \& Westenberg, P. M. (2007). Peer behavior toward socially anxious adolescents: Classroom observations. Behaviour Research and Therapy, 45, 2773-2779. 
Martins, A. C., Almeida, J. P. \& Viana, V. (2014). Ansiedade Social na Infância e Pré-Adolescência: Adaptação para o Português de Portugal da SASC-R.

Bradley, C (1996). Translaction of questionnaires for use in different languages and cultures. In C. Bradley (Ed.), Handbook of psychology and diabetes. A guide to psychological measurement in diabetes research and practice. London: Harwood Academic.

Casas, C., Álvarez, M., Lopez, L., \& Aranda, B. (2012). Adaptación transcultural de un tratamiento para la fobia social: un estudio piloto. International Journal of Psychology and Psychological Therapy, 12(1), 35-48.

Clark, D., Turner, S., Beidel, D., Donovan, J., Kirisci, L., \& Jacob, R. (1994). Reliability and validity of the Social Phobia and Anxiety Inventory for Adolescents. Psychological Assessment, 6, 135-140.

Cole, D. (1987). Methodological contributions to clinical research: Utility of confirmatory factor analysis in test validation research. Journal of Consulting and Clinical Psychology, 55(4), 584-594.

Connor, K. M., Davidson, J. R., Churchill, L. E., Sherwood, A., Foa, E., \& Weisler, R. H. (2000). Psychometric properties of the Social Phobia Inventory (SPIN). New self-rating scale. British Journal of Psychiatry, 176, 379-386.

Cunha, M., \& Pinto Gouveia, J. (1997). Avaliação clínica da fobia social. Psiquiatria Clínica, 18(4), 313-331.

Cunha, M., Pinto Gouveia, J., Alegre, S., \& Salvador, M. C. (2004). Avaliação da ansiedade na adolescência: A versão portuguesa da SAS-A. Psychologica, 35, 249-263.

Cunha, M., Pinto Gouveia, J., Salvador, M. C., \& Alegre, S. (2004). Medos sociais na adolescência: A Escala de Ansiedade e Evitamento de Situações Sociais para Adolescentes (EAESSA). Psychologica, 36, 195-217.

Cunha, M., \& Salvador, M. (2000). Fobia social na infância e adolescência: Diagnóstico, avaliação e tratamento. In J. P. Gouveia (Ed.), Ansiedade social: Da timidez à fobia social. Coimbra, Portugal: Quarteto.

Garcia-López, L. J., Olivares, J., Hidalgo, M. D., Beidel, D. C., \& Turner, S. M. (2001). Psychometric properties of the Social Phobia and Anxiety Inventory, the Social Anxiety Scale for Adolescents, the fear of Negative Evaluation Scale and the Social Avoidance Distress Scale in an Adolescent Spanish Speaking Population. Journal of Psychopathology and Behavioral Assessment, 23, 51-59.

Greco, L. A., \& Morris, T. L. (2005). Factors influencing the link between social anxiety and peer acceptance: Contributions of social skills and close friendships during middle childhood. Behavior Therapy, 36, 197-205.

Harter, S. (1982). The perceived competence scale for children. Child Development, 53, 87-97.

Inderbitzen, H. M., \& Foster, S. (1992). The Teenage Inventory of Social Skills: Development, reliability, and validity. Psychological Assessment, 4, 451-459.

Ito, L., Roso, M., Tiwari, S., Kendall, P., \& Asbahr, F. (2008). Terapia cognitivo-comportamental da fobia social. Revista Brasileira de Psiquiatria, 30, 96-101.

Johnson, H. S., Inderbitzen-Nolan, H. M., \& Anderson, E. R. (2006). The Social Phobia Inventory: Validity and reliability in an adolescent community sample. Psychological Assessment, 18, 269-277.

Kuusikko, S., Pollock-Wurman, R., Ebeling, H., Hurtig, T., Joskitt, L., Mattila, M., ...Moilanen, I. (2009). Psychometric evaluation of Social Phobia and Anxiety Inventory for Children (SPAI-C) and Social Anxiety Scale for Children-Revised (SASC-R). European Child \& Adolescent Psychiatry, 18(2), 116-124.
La Greca, A. M., \& Lopez, N. (1998). Social anxiety among adolescents: Linkages with peer relations and friendships. Journal of Abnormal Child Psychology, 26, 83-94.

La Greca, A. M., \& Stone, W. (1993). Social Anxiety Scale for Children-Revised: Factor structure and concurrent validity. Journal of Clinical Child Psychology, 22, 17-27.

Matson, J., Rotatori, A., \& Helsel, W. (1983). Development of a Rating Scale to Measure Social Skills in Children: The Matson Evaluation of Social Skills with Youngsters (MESSY). Behaviour Research and Therapy, 21, 335-340.

Moore, K. A., \& Gee, D. L. (2003). The reliability, validity, discriminant and predictive properties of the Social Phobia Inventory (SoPhI). Anxiety, Stress, and Coping, 16(1), 109-117.

Olivares, J., Garcia-López, L. J., Hidalgo, M. D., Turner, S. M., \& Beidel, D. C. (1999). The Social Phobia and Anxiety Inventory: Reliability and validity in an adolescent Spanish population. Journal of Psychopathology and Behavioral Assessment, 21, 67-78.

Oreskong, K., \& Sorbom, D. (1993). Lisrel 8 user's reference guide. Chicago, IL: Science Software International.

Ost, L. (2008). Cognitive behavior therapy for anxiety disorders: 40 years of progress. Nordic Journal of Psychiatry, 62, 5-10.

Pinto Gouveia, J. (2000). Ansiedade social: Da timidez à fobia social. Coimbra, Portugal: Quarteto.

Reijntjes, A., Dekovic, M., \& Telch, M. (2007). Support for the predictive validity of the SASC-R: Linkages with reactions to an in vivo peer evaluation manipulation. Journal of Anxiety Disorders, 21(7), 903-917.

Salvador, M. (2009). Ser eu próprio entre os outros: Um novo protocolo de intervenção para adolescentes com fobia social generalizada (Tese de doutorado, Universidade de Coimbra, Coimbra, Portugal).

Sandín, B., Chorot, P., Valiente, R., Santed, M., \& Sánchez-Arribas, C. (1999). Estructura factorial de la Escala de Ansiedade Social para Ninos-Revisada (SASC-R). Revista de Psicopatologia y Psicología Clínica, 4(2), 105-113.

Silveira, D., \& Valença, A. (2005). Fobia social: Clínica e tratamento. Arquivos Brasileiros de Psiquiatria, Neurologia e Medicina Legal, 99(2), 21-25.

Stein, M. B., \& Kean, Y. M. (2000). Disability and quality of life in social phobia: Epidemiologic findings. American Journal of Psychiatry, 157, 1606-1613.

Storch, E. A., \& Masia-Warner, C. (2004). The relationship of peer victimization to social anxiety and loneliness in adolescent females. Journal of Adolescence, 27, 351-362.

Toit, P., \& Stein, D. (2003). Social anxiety disorder. In D. Nutt \& J. Ballenge (Eds.), Anxiety disorders (pp. 39-50). Malden, MA: Blackwell.

Van Ameringen, M., Mancini, C., \& Farvolden, P. (2003). The impact of anxiety disorders on educational achievement. Journal of Anxiety Disorders, 17, 561-571.

Warren, R., Good, G., \& Velten, E. (1984). Measurement of social-evaluative anxiety in junior high school Students. Adolescence, 19, 643-648.

Watson, D., \& Friend, R. (1969). Measurement of social-evaluative anxiety. Journal of Consulting and Clinical Psychology, 33, 448-457.

Recebido: 23/11/2011

$1^{a}$ revisão: $14 / 01 / 2013$

Aceite final: 05/03/2013 